\title{
Análise comparativa dos benefícios do Taekwondo na flexibilidade em crianças e adolescentes praticantes e não praticantes: Revisão de literatura
}

Analysis of the Taekwondo on practicing and non-practicing children and adolescent's flexibility:

\section{Literature review}

Análisis del Taekwondo sobre la flexibilidad de niños y adolescentes practicantes y no practicantes:

\section{Revisión de literatura}

Peters Ribeiro de Oliveira

ORCID: https://orcid.org/0000-0002-9611-9078 Centro Universitário Augusto Motta, Brasil

E-mail: peters.ribeiro.oliveira@gmail.com

Jeniffer Cristina Cardoso Goehring Vital

ORCID: https://orcid.org/0000-0002-8675-8917 Centro Universitário Augusto Motta, Brasil

E-mail: crisgoehring10@gmail.com

Leonardo Nascimento de Oliveira ORCID: https://orcid.org/0000-0002-2320-3420 Centro Universitário Augusto Motta, Brasil

E-mail: lee.oliveira2014@gmail.com

Zenilda Seixas Figueredo

ORCID: https://orcid.org/0000-0001-9903-1138 Centro Universitário Augusto Motta, Brasil

E-mail: zenildafigueredo32@gmail.com

Rudson Santos da Silva

ORCID: https://orcid.org/0000-0002-8859-985X

Fundação Oswaldo Cruz, Brasil

E-mail:rss1917@gmail.com

Victor Gonçalves Corrêa Neto

ORCID: https://orcid.org/ 0000-0003-3133-1630

Centro Universitário Gama e Souza, Brasil

Universidade Estácio de Sá, Brasil

E-mail: victorgen@hotmail.com

Estêvão Rios Monteiro

ORCID: https://orcid.org/0000-0003-1866-553X

Centro Universitário Augusto Motta, Brasil

Universidade Federal do Rio de Janeiro, Brasil

E-mail: profestevaomonteiro@gmail.com

Marcelo José Colonna de Miranda ORCID: https://orcid.org/0000-0003-1552-9153

Centro Universitário Augusto Motta, Brasil

Universidade Estácio de Sá, Brasil

E-mail: marcelocolonna71@gmail.com

\section{Resumo}

O objetivo do presente estudo foi revisar as evidências sobre os benefícios do Taekwondo na flexibilidade em crianças e adolescentes praticantes e não praticantes. Foi realizada uma busca na base de dados do Google Acadêmico, sendo incluídos artigos publicados entre 2010 e março de 2021 e usando como descritores: "Taekwondo", "flexibilidade", "lutas e artes marciais", e "amplitude de movimento". Foram incluídos estudos originais, desde que redigidos na língua portuguesa. Foram incluídos três artigos, os quais majoritariamente tecem informações positivas sobre a prática do Taekwondo na melhora da flexibilidade. Os resultados também revelam que alunos praticantes apresentam melhoras expressivas na variável flexibilidade quando comparados aos não praticantes. Assim, o Taekwondo promove o aumento da flexibilidade, já que em suas principais técnicas empregadas utilizam-se chutes, movimento do quadril, mãos, que contribuem para melhor flexibilidade.

Palavras-chave: Amplitude de movimento articular; Artes marciais; Criança; Medicina esportiva.

\section{Abstract}

The purpose of the present study was to review the evidence on the benefits of Taekwondo on flexibility in practicing and non-practicing children and adolescents. A search was performed throughout Google Scholar database, including 
articles published between 2010 and March 2021 and using as descriptors: 'taekwondo', 'flexibility', 'range of motion', and 'martial arts'. Original research was included and need to write in Portuguese. Three articles were included, which mostly weave positive information about Taekwondo and flexibility. Additionally, students who practice Taekwondo show improvements in flexibility when compared to non-practitioners. Thus, Taekwondo promotes increased flexibility, since the main techniques used use kicks, hip movement, hands, which contribute to better flexibility.

Keywords: Range of joint motion; Martial arts; Child; Sports medicine.

\section{Resumen}

El objetivo del presente estudio fue revisar la evidencia sobre los beneficios del Taekwondo sobre la flexibilidad en niños y adolescentes practicantes y no practicantes. Se realizó una búsqueda en la base de datos de Google Scholar, incluyendo artículos publicados entre 2010 y marzo de 2021 y utilizando como descriptores: 'Taekwondo', 'flexibilidad', 'rango de movimiento' y 'artes marciales'. Se incluyó la investigación original y es necesario escribirla en portugués. Se incluyeron tres artículos, que en su mayoría tejen información positiva sobre Taekwondo y flexibilidad. Además, los estudiantes que practican Taekwondo muestran mejoras en la flexibilidad en comparación con los no practicantes. Así, el Taekwondo promueve una mayor flexibilidad, ya que las principales técnicas empleadas son patadas, movimiento de cadera, manos, que contribuyen a una mejor flexibilidad.

Palabras clave: Rango de movimiento articular; Artes marciales; Niño; Medicina deportiva.

\section{Introdução}

O Taekwondo é uma arte marcial que abrange diversos movimentos corporais que colaboram para o processo da aprendizagem motora, geralmente praticado em academias de luta, adotando um processo de aprendizado organizado, através de repetição de movimentos coordenados, que se configuram como partes características da aula (Costa et al., 2012). Costa et al. (2012) mencionam a importância da prática do Taekwondo para as crianças, tendo em vista de que o mesmo é de grande relevância para o período infantil, por ser uma etapa que se iniciam as relações interambientais e interpessoais. Logo, quanto mais enriquecido for o ambiente em que a criança interage, as possibilidades de desenvolvimento pessoal poderão acontecer, além de melhor nível de amadurecimento dos sistemas corporais. Para Vygotsky (2007) o desenvolvimento humano não é determinado apenas por processos de maturação biológicos, mas está atrelado à apropriação da cultura, envolvendo diversos campos da existência, tais como: afeto, cognitivo e social. Corroborando o que foi dito, Rufino e Darido (2015) acrescentam que o desenvolvimento humano é o movimento entre o espontâneo e o intencional, compreendendo diversos contextos do desenvolvimento humano, seja ele psicomotor, psicossocial, psicocomportamental, cultural ou filosófico.

Segundo a Base Nacional Comum Curricular (BNCC) a criança tem o direito de expressar, como sujeito criativo, dialógico e sensível, suas emoções, necessidades, dúvidas, sentimentos, descobertas, hipóteses, questionamentos e opiniões novamente, por meio de diferentes linguagens (BRASIL, 2017). Ainda segundo a BNCC, alguns eixos representam importantes componentes para a Educação Física escolar, dentre eles, destacam-se as lutas e artes marciais, pois são meios para o desenvolvimento de competências, abrangendo os aspectos físicos, socioculturais e o desenvolvimento do educando, Ressalte-se que, a partir do $6^{\circ}$ ano, prevê-se que os estudantes possam ter acesso a um conhecimento mais aprofundado de algumas das práticas corporais, como também sua realização em contextos de lazer e saúde, dentro e fora da escola. (BRASIL, 2017).

Para Willirich, Azevado e Fernandes (2009) o desenvolvimento motor é considerado como um processo sequencial, consecutivo e conexo à idade cronológica, em que o sujeito adquire grande quantidade de habilidades motoras, as quais avançam de movimentos simples e desorganizados para a execução de habilidades motoras altamente organizadas e complexas. O surgimento de movimentos e seu posterior controle acontecem em uma direção céfalo-caudal e próximo-distal. Entretanto, este processo não se apresenta de forma linear, abrangendo períodos de equilíbrio e desequilíbrio. Apesar disso, costuma cumprir uma sequência ordenada e até previsível de acordo com a idade. Tozetto et al. (2012) indicam que no processo de treinamento com as crianças devem ser priorizados, tendo como objetivos o desenvolvimento das qualidades físicas, a formação dos hábitos motores e a assimilação dos conhecimentos, o que pode proporcionar à criança utilizar com 
êxito os ganhos da cultura física e do desporto nas atividades da vida diária. Com isso, o desenvolvimento da força junto à flexibilidade tem grande importância, pois possibilita tempos de duração maiores na sustentação da postura, dependendo da capacidade de gerar força.

Dantas (2014) enfatiza que a flexibilidade é a capacidade de executar os movimentos articulares com maior amplitude possível sem que aconteçam danos às articulações. Ela é característica para cada exercício. Igualmente, a agilidade diz respeito à capacidade de mudar de direção ligeiramente, ela atrela-se à velocidade e força, sendo muito empregada nos esportes coletivos. Ainda, Dantas (2017) complementa que a flexibilidade configura se como a qualidade física responsável pela execução voluntária de um movimento de amplitude angular máxima, ainda por uma articulação ou conjunto de articulações, dentro das limitações morfológicas, sem que haja eventuais riscos de provocar lesões. Araújo e Oliveira (2008) não observaram diferenças na flexibilidade para ambos os sexos com o avanço da idade (10 a 14 anos), recomendando, de tal modo, que a idade não está correlacionada com a flexibilidade na infância e adolescência.

Segundo Vieira e Roman (2020) as práticas constantes de exercícios de flexibilidade contribuem para redução de lesões. A insuficiência da flexibilidade contribui para o desenvolvimento de lesões facilmente e limite da amplitude de movimentos para execução de técnicas complexas, com redução da qualidade dos golpes de perna na altura da cabeça. Para o treinamento da flexibilidade deve-se considerar a idade biológica e cronológica, força muscular, nível de treinamento e combinação das medidas organizadas de treinamento (ativa, passiva e mista). Venson (2014) destaca que as lutas trazem numerosos benefícios ao praticante no que diz respeito ao desenvolvimento motor, afetivo, cognitivo e social. Destaca-se na questão motora a lateralidade, o controle do tônus muscular, o equilíbrio, a organização global, a ideia de tempo e espaço e a noção de corpo; no aspecto cognitivo, a inteligência, o entendimento, a formulação de táticas e a atenção; e finalmente, no contexto social verificam-se nos alunos alguns aspectos admiráveis como a reação a verificadas atitudes, a postura social, a constância, o respeito e a determinação, além de beneficiar a criança a desenvolver o sentido do tato, extravasar e controlar a agressividade, aumentar a responsabilidade.

Resende et al. (2016), em seus estudos constataram que as atividades físicas fora da escola cooperam para o desenvolvimento das valências físicas relacionada ao esporte. Já Costa et al. (2012) constatou que a escola como espaço educacional e, deste modo, de aprendizagem, encontra-se em constante processo de mudanças, além de ter a função de promover mudanças nos alunos, elas podem ser tanto no aspecto cognitivo, quanto motor. Para Costa et al. (2012), o motivo principal do aprendizado motor é contribuir para o aumento da capacidade do sujeito no desempenho com sucesso de determinada habilidade e para isso, requer o emprego ordenado de múltiplos músculos e articulações do corpo humano. Ao avaliar a aprendizagem, o docente de Educação Física deverá considerar o desempenho apresentado pelo aluno, compreendendo que as ações praticadas deste aprendizado poderão ser transferidas de modo que beneficie outras habilidades.

Vieira e Romam (2020) a prática do Taekwondo no âmbito contribuirá para as crianças com o emprego de gestos motores diversos, que poderão ser empregados para facilitar o aprendizado motor. Costa et al. (2012) destacam que os movimentos do Taekwondo auxiliam como parâmetros de avaliação dos praticantes no decorrer da troca de faixa. De tal modo, ao aferir o aprendizado, deve-se considerar à prática constante desta modalidade. Deve-se levar em consideração, porém, que o Taekwondo é um desporto que exige um alto grau de flexibilidade, pois, como sugere Lima (1993), isto permite que o lutador execute um chute com grande alcance com menor esforço, consequentemente com menor dispêndio de energia e maior eficácia técnica. Vieira e Roman (2020) acrescentam que para treinamento do Taekwondo é imprescindível que os praticantes realizem exercícios para a flexibilidade, com destaque para os membros inferiores, pois é uma luta que envolve chutes altos, e com boa flexibilidade será possível alcançar a realização de diversos movimentos amplos com maior velocidade, o que é eficaz para a luta. Ao se relacionar com o próprio corpo, como meio social e com o outro, o educando desenvolve a convivência social, 
respeito às diferenças e aprende novas culturas que essas práticas, de um modo geral, que promovem vivências que facilitarão seu aprendizado, além de contribuir para o seu desenvolvimento pleno.

Dessa forma, o objetivo do presente estudo foi revisar as evidências sobre os benefícios do Taekwondo na flexibilidade em crianças e adolescentes praticantes e não praticantes.

\section{Metodologia}

\subsection{Tipo de estudo}

O estudo em tela é uma pesquisa de cunho de revisão de literatura (Estrela, 2018).

\subsection{Seleção dos artigos}

A busca foi feita na base de dados do Google Acadêmico, sendo incluídos artigos publicados entre 2010 e março de 2021. Durante a pesquisa, descritores como "Taekwondo", "flexibilidade", "lutas e artes marciais", e "amplitude de movimento" foram utilizados nos campos de busca das bases de dados. A Figura 1 trás uma síntese das etapas de seleção dos artigos.

Figura 1. Flowchart do estudo.

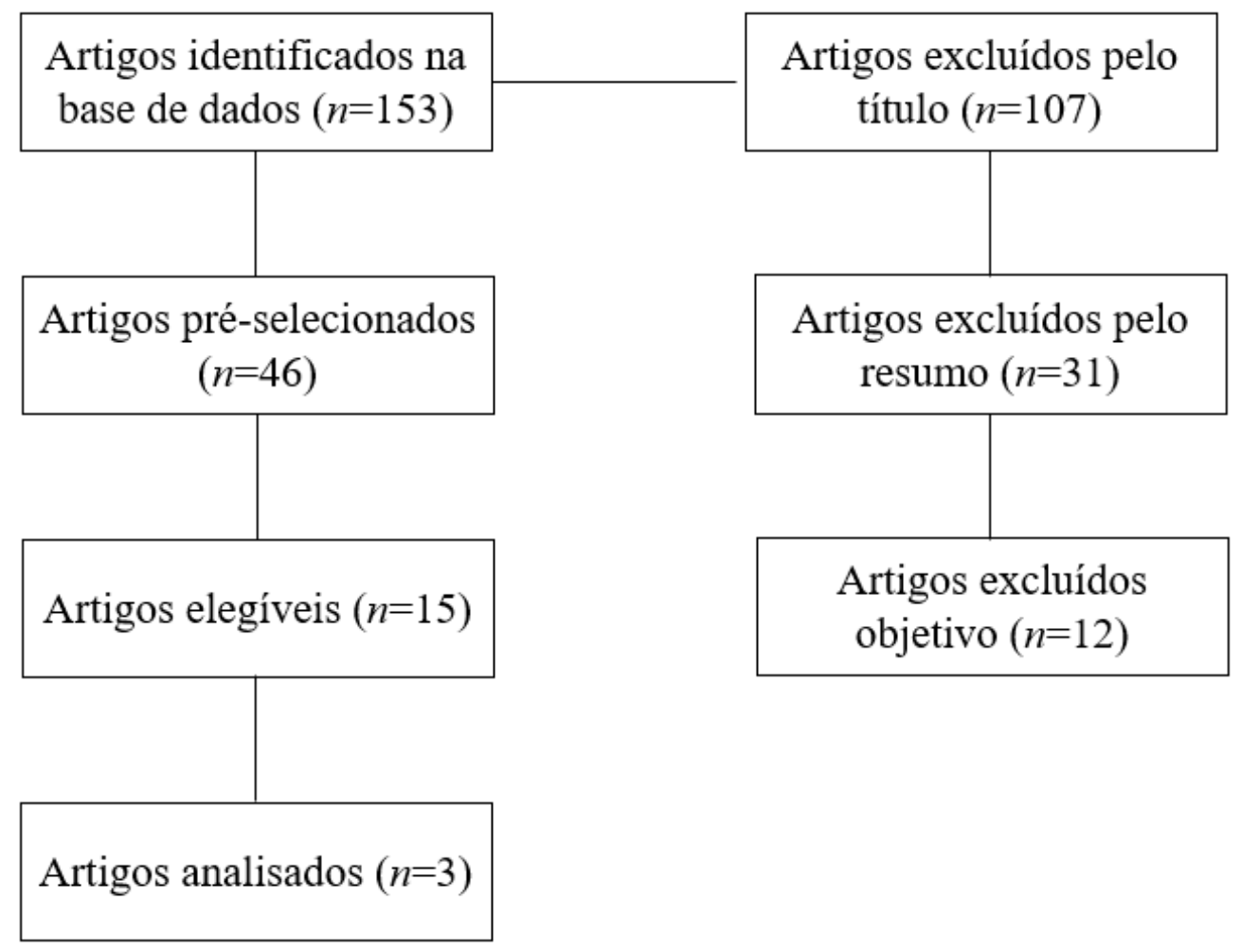

Fonte: Autores.

Os estudos encontrados com essas palavras-chaves foram avaliados e selecionados de acordo com os seguintes critérios de inclusão: a) artigo original e b) estar redigido na língua portuguesa. Foram descartados artigos que tenham realizados intervenção médica. 
Os resumos dos artigos foram lidos para que os preceitos elencados acima fossem aplicados. Selecionando assim, os artigos que se destinaram a ser lidos na íntegra. Os artigos selecionados têm como aplicabilidade a avaliação da flexibilidade em crianças e adolescentes praticantes e não praticantes de Taekwondo.

\section{Resultados e Discussão}

A Figura 1 traz o flowchart indicando todas as etapas da busca. Foram encontrados um total de 153 artigos e, após passarem pelos critérios de inclusão e exclusão, foram analisados 3 artigos no total: Costa, Silva e Gheller (2015), Silva et al. (2017) e Pinto et al. (2018). A Tabela 1 traz a síntese das principais informações extraídas dos estudos.

Tabela 1 - Descrição dos estudos selecionados.

\begin{tabular}{|c|c|c|c|c|}
\hline Autor/Ano & TÍTULO & Amostra & Protocolo & Conclusão \\
\hline $\begin{array}{l}\text { Pinto et al. } \\
(2018)\end{array}$ & $\begin{array}{l}\text { Adolescentes praticantes e } \\
\text { não praticantes de } \\
\text { taekwondo: uma análise } \\
\text { comparativa } \\
\text { flexibilidade e agilidade }\end{array}$ & $\begin{array}{l}\text { Os participantes de ambos } \\
\text { os gêneros (n=40) } \\
\text { possuíam idade entre } 12 \text { e } \\
15 \text { anos, foram divididos } \\
\text { em "grupo teste" }(\mathrm{n}=20) \text { e } \\
\text { "grupo controle" não } \\
\text { praticantes de Taekwondo } \\
(\mathrm{n}=20) \text {. }\end{array}$ & $\begin{array}{l}\text { Foi promovido um protocolo de } \\
\text { treinamento com a durabilidade } \\
\text { de } 12 \text { semanas (três vezes na } \\
\text { semana com duração de } 2 \text { horas } \\
\text { cada seção), cada seção foi } \\
\text { estruturada em três etapas: i) } \\
\text { aquecimento (alongamentos); } \\
\text { ii) técnicas da arte marcial } \\
\text { (chutes, combates, poomses); } \\
\text { iii) (alongamento) }\end{array}$ & $\begin{array}{l}\text { O Taekwondo contribuiu para } \\
\text { melhora dos níveis das valências } \\
\text { físicas: flexibilidade e agilidade } \\
\text { em ambos os gêneros estudados no } \\
\text { grupo testem. }\end{array}$ \\
\hline $\begin{array}{l}\text { Silva et al. } \\
(2017)\end{array}$ & $\begin{array}{l}\text { Análise comparativa da } \\
\text { flexibilidade, agilidade e } \\
\text { força de membros } \\
\text { inferiores em adolescentes } \\
\text { praticantes e não } \\
\text { praticantes de Taekwondo }\end{array}$ & $\begin{array}{l}\text { Os participantes de ambos } \\
\text { os gêneros } \quad(\mathrm{n}=60) \\
\text { possuíam idade entre } 11 \text { e } \\
14 \text { anos }\end{array}$ & $\begin{array}{l}\text { Protocolo de treinamento com a } \\
\text { durabilidade de } 12 \text { semanas } \\
\text { (três vezes na semana com } \\
\text { duração de } 2 \text { horas cada seção), } \\
\text { cada seção foi estruturada em } \\
\text { três etapas: i) aquecimento } \\
\text { (alongamentos); ii) técnicas da } \\
\text { arte marcial (chutes, combates, } \\
\text { poomses); iii) (alongamentos). }\end{array}$ & $\begin{array}{l}\text { O Taekwondo contribuiu para } \\
\text { melhora dos níveis das valências } \\
\text { físicas: flexibilidade, agilidade e } \\
\text { força de membros inferiores em } \\
\text { ambos os gêneros estudados no } \\
\text { grupo testem }\end{array}$ \\
\hline $\begin{array}{l}\text { Costa, Silva e } \\
\text { Gheller (2015 }\end{array}$ & $\begin{array}{lll}\text { Influência da } & \text { prática } & \text { do } \\
\text { Taekwondo } & & \text { na } \\
\text { flexibilidade } & & \text { de } \\
\text { adolescentes } & & \end{array}$ & $\begin{array}{lrr}\text { A amostra foi composta } \\
\text { por } 15 \text { adolescentes que } \\
\text { praticavam } & \text { e } & 15 \\
\text { adolescentes } & \text { que } & \text { não } \\
\text { praticavam } & \text { Taekwondo, } \\
\text { com idade entre } 11 \text { e } 13 \\
\text { anos }\end{array}$ & $\begin{array}{l}\text { Teste t de Student para } \\
\text { amostras independentes. }\end{array}$ & $\begin{array}{l}\text { O Taekwondo é uma modalidade } \\
\text { esportiva que melhora os níveis de } \\
\text { flexibilidade de todos os seus } \\
\text { praticantes. }\end{array}$ \\
\hline
\end{tabular}

Fonte: Autores.

O objetivo do presente estudo foi revisar as evidências sobre os benefícios do Taekwondo na flexibilidade em crianças e adolescentes praticantes e não praticantes. A prática do Taekwondo contribui para melhor flexibilidade consequentemente melhora agilidade, equilíbrio, autoconfiança e autodefesa. Neste sentido, Pinto et al. (2018) analisaram a Flexibilidade dos inquiridos no estudo através do teste de sentar e alcançar (avaliação da cadeia posterior) encontrando melhora no grupo em ambos os gêneros, entretanto, o grupo experimental masculino alcançou um resultado mais expressivo de ganho de flexibilidade quando comparado ao pré-treino para o pós-treino, com variação de 4,57 centímetros de ganho. Pinto et al. (2018) em um estudo realizado com aplicação de um protocolo de lutas durante 16 semanas em 28 escolas, foi possível observar melhora expressiva na valência Flexibilidade depois do período de prática. Os resultados mostraram melhoras de $85 \%$ dos meninos e 95\% das meninas melhoraram na valência Flexibilidade, confirmando os resultados do estudo de Ferreira (2008).

Pinto et al. (2018) chamam atenção para outro estudo realizado com jovens praticantes de lutas, sendo possível observar um aumento significativo na flexibilidade, onde Marinho et al. (2011) constataram que neste tipo de modalidade, há uma grande realização das técnicas que exigem maior amplitude de movimento, contribuindo para o grau de Flexibilidade. Silva et al. (2017) chegaram ao resultado de que alunos praticantes de Taekwondo, do gênero feminino, as variáveis 
flexibilidade, agilidade e força de membros inferiores alcançaram expressivas melhoras quando comparadas com a fase pré para a pós prática. Para o gênero masculino constatou-se também melhoras expressivas das variáveis avaliadas, flexibilidade, agilidade e força dos membros inferiores, comparando a fase pré para a pós-prática. Um estudo com resultados semelhantes ao de Silva et al. (2017), realizado por 12 semanas de prática regular da Taekwondo, foi exposto diferenças nos níveis de Flexibilidade, quando confrontado ao grupo de controle. Tal pressuposto pode ser explicado em detrimento dos movimentos empregados nas técnicas de Taekwondo exigir muito dessa variável físicas Silva et al. (2017).

Dentre as capacidades mais utilizadas nas aulas de Taekwondo, destaca-se a flexibilidade. Costa, Silva e Gheller (2015) ressaltam que flexibilidade configura se como a capacidade de alongar, dobrar ou torcer determinada parte do corpo, dependendo essencialmente da musculatura, tendões e articulações e quanto maior a frequência e intensidade do alongamento, mais rápido será o resultado de uma boa flexibilidade. Com o mesmo entendimento, Achour Júnior (1998) reforçam que a estrutura de programas de flexibilidade, deve ser implementada com exercícios de alongamento, a fim de eliminar ou impedir eventuais lesões ou encurtamentos musculares oriundos de esforços durante a realização dos exercícios ou ainda para mitigar tensões musculares.

Costa, Silva e Gheller (2015) ressaltam que para determinados esportes ou atividades físicas que demandam maior flexibilidade do que outros para a execução de sua modalidade ou mesmo melhora do desempenho físico, a flexibilidade apresenta especificidade em relação à atividade física nas distintas articulações utilizadas para determinadas práticas desportivas. Além de colaborar para uma técnica mais acurada, com aumento da eficiência e melhor segurança do gesto motor. Costa, Silva e Gheller (2015) aplicaram o Flexiteste adaptado, à fim de avaliar a mobilidade de 08 movimentos corporais, como: tronco, articulações do quadril e ombro, sem o aquecimento prévio. Os movimentos foram avaliados em uma escala de zero a quatro, com cinco níveis de flexibilidade. O teste foi aplicado por um professor, que forçou a movimentação nas articulações de 15 adolescentes avaliados, até alcançar o ponto máximo de amplitude, prontamente detectado em função do desconforto do mesmo. Os autores chegaram ao resultado de os adolescentes praticantes tiveram um nível de Flexibilidade, muito grande (hipermobilidade). Entretanto, os nãos praticantes obtiveram um nível de Flexibilidade, médio negativo segundo a tabela do Flexiteste (Costa; Silva e Gheller, 2015).

Segundo Fonseca (2004) o treinamento da flexibilidade é imprescindível para o Taekwondo, pois, o desenvolvimento de altos níveis de mobilidade articular do tronco e dos membros inferiores, contribui para um melhor desenvolvimento da capacidade de executar chutes. Assim sendo, Costa, Silva e Gheller (2015) ratificam que a flexibilidade, como legalidade específica, relaciona-se de maneira positiva a prática de Taekwondo e ainda haverá melhor desenvolvimento da técnica e tática de seus praticantes. Desta forma, o Taekwondo pode colaborar para melhor flexibilidade, proporcionando as crianças praticantes desta modalidade, uma vida cheia de conquistas tanto no âmbito físico como no cognitivo.

\section{Considerações Finais}

O presente estudo concluiu que a prática do Taekwondo beneficia a flexibilidade dos alunos praticantes. Os estudos ainda revelaram que alunos praticantes apresentam melhoras expressivas na variável flexibilidade quando comparados aos não praticantes. Assim, o Taekwondo promove o aumento da flexibilidade, já que em suas principais técnicas empregadas utilizamse chutes, movimento do quadril, mãos, que contribuem para melhor flexibilidade. Assim sendo, os autores entendem a importância que o Taekwondo trás para o desenvolvimento motor de crianças e adolescentes, mas também entendem a necessidade de estudos futuros a fim de observar melhor esses efeitos, bem como efeitos adicionais em outras valências físicas. Dessa forma, os autores sugerem a realização de novos estudos testando diferentes desenhos experimentais e comparando o Taekwondo com outras modalidades esportivas. 


\section{Agradecimentos}

Os autores agradecem a participação de todos os voluntários dessa pesquisa.

\section{Referências}

Araújo, S. S.; Oliveira, A. C. C. (2008). Aptidão física em escolares de Aracaju. Rev Bras Cineantropom Desempenho Hum, 10 (3), $271-276$.

Achour Júnior, A. (1998). Bases para exercícios de alongamento relacionado com a saúde e no desempenho atlético. Editora Midiograf.

Brasil. Ministério da Educação. Secretaria da Educação Básica. Base Nacional Comum Curricular. Brasília-DF; MEC; CONSED; UNDIME, 2017.

Costa, O. M.; Santos, V. F. (2012). O Taekwondo e sua contribuição na educação infantil: um estudo lúdico. EFDeportes, 17 (167), 1-6.

Costa, E. L.; Silva, R. B.; Gheller, R. G. (2015). Influência da prática do Taekwondo na flexibilidade de adolescentes. EFDeportes, 20 (206), 1-6.

Dantas, E. H. M. (2014). A Prática da Preparação Física. Editora Roca.

Dantas, E. H. M. (2017). Flexibilidade, alongamento e flexionamento. Editora Manole.

Ferreira, J. S.; Ledesma, N. C. (2008). Indicadores de flexibilidade em escolares de 11 anos de idade de uma escola de Campo Grande-MS, Brasil. EFDeportes, 12 (118), 10-18.

Fonseca, V. (2004). Psicomotricidade: perspectivas multidisciplinares. Editora Artmed.

Lima, A. A. (1993). Flexibilidade no Taekwondo. Editora Biopress.

Marinho, B. F.; Del Vecchio, F. B.; Frachini, E. (2011). Condicion física y perfil antropométrico de atletas de artes marciales. Revista de artes Marciales Asiáticas, $6(2), 1-12$.

Nunez, A. M. G. (2005). Algunas consideraciones acerca del entrenamiento de la flexibilidad en el taekwondo. EFDeportes, 10 (87), 1-6.

Pinto, B. A. P.; Da Silva, D. R.; Ferreira, D. S.; Apolinário, K. A. P.; Resende, L. A. P.; Caetano, L. F.; Marques, S. A.; Da Silva, G. R.; Reis, A. M. (2018). Adolescentes praticantes e não praticantes de Taekwondo: uma análise comparativa da flexibilidade e agilidade. Revista Gestão Universitária, 10 (2), 1-8.

Resende, F. N.; Costa, W. R.; Fidale, T. M.; Agostini, G. G.; Marcacini, J. P.; Lana, R. P.; Marocolo, M. (2016). Comparação da força de tronco, resistência abdominal e potência de membro superior e inferior de alunos de $5^{\mathrm{a}}$ a $8^{\mathrm{a}}$ série do ensino fundamental de escola pública e particular. Revista Arquivo de Ciência do Esporte, 4 (1), 10-15.

Rufino, L. G. B.; Darido, S. C. (2015). O ensino das lutas nas aulas de educação física: análise da prática pedagógica à luz de especialistas. Rev Educ Fís/UEM, 26 (4), 505-518.

Silva, J. F.; Silvestre, T. F.; Silva, G. R.; Terra, G. D. S. V.; Neiva, C. M.; Tavares, M. R.; Boas, Y. F. V.; Rodrigues, C. A. C. (2017). Análise comparativa da flexibilidade, agilidade e força de membros inferiores em adolescentes praticantes e não praticantes de Taekwondo. Conexão $\mathrm{Ci}$, 12 (3), 7-16.

Tozetto, A. V. B.; Milistetd, M.; Medeiros, T. E.; Ignachewski, W. L. (2012). Desempenho de Jovens Atletas Sobre as Capacidades Físicas, Flexibilidade, Força e Agilidade. Cinergis, 13 (2), 47-54.

Venson, G. E.; Graça, R. L. (2014). Motivos que levam os professores a (des) considerarem a luta como conteúdo de educação física escolar. EFDeportes, 19 (198), 1-10

Willirich, A.; Azevedo, C. C. F.; Fernandes, J. O. (2009). Desenvolvimento Motor na Infância: Influência dos Fatores de Risco e Programas de Intervenção. Rev Neurocienc, 7 (1), 51-56.

Vygotsky, L. (2007). A formação social da mente. Editora Martins Fontes. 